\title{
Producción y comercialización de rosquillas Delicias del Norte en la ciudad de Somoto Madriz. Del I semestre del año 2013 al II semestre del año 2014
}

\author{
Daniela Lisbeth Martínez \\ Indira Yomaris Cruz Bustamante ${ }^{1}$ \\ Dana Iveth López Altamirano \\ Beverly Castillo Herrera ${ }^{2}$
}

\section{RESUMEN}

En el presente estudio se analizó el proceso de producción y comercialización de la empresa de rosquillas Delicias del Norte del municipio de Somoto en el periodo 2013- 2014. Esta investigación es de tipo cualitativa por que se describe y analiza a profundidad el proceso de producción y comercialización de las rosquillas somoteñas Delicias del norte, para ello se hace una descripción detallada y se analiza exclusivamente el problema para proponer una estrategia que mejore el proceso de comercialización de la empresa. El universo de estudio está conformado por la empresa Delicias del Norte que cuenta con un total de 20 trabajadores incluyendo el área administrativa. Dentro de las mejoras que se deben hacer en Delicias del Norte está el aprovechamiento de la capacidad productiva tomando en cuenta que posee todos los requisitos necesarios para aumentar su producción, en el proceso de comercialización se propone mejorar el proceso de comercialización mediante una mayor inversión en el material publicitario.

Palabras Clave: Proceso, Producción, Comercialización.

Recibido: 15 de diciembre de 2014

Aceptado: 22 de junio de 2015

1 UNAN-Managua/FAREM-Estelí. Correo Electrónico: indicruz_15@yahoo.es

2 Candidata a doctora en Ciencias Sociales por la Universidad del Zulia. Maestría en Ciencias Sociales por la Universidad de Guadalajara. Docente titular de la UNAN-Managua, FAREM-Estelí. Correo Electrónico:

beverly.castillo@yahoo.com. 


\title{
Production and marketing of Rosquillas Delicias del Norte in the city of Somoto Madriz. From the first semester of the year 2013 to the second semester of the year 2014
}

\begin{abstract}
In this study the production process and marketing of the company Rosquillas Delicias del Norte of the town of Somoto was analyzed in the period 2013-2014. This research is qualitative type since it describes and analyzes in depth the production and marketing process of Somoto Rosquillas Delicias del Norte, for which a detailed description is made and the problem is analyzed exclusively to propose a strategy to improve the marketing process of the company. The study group consists of the company Delicias del Norte which has a total of 20 employees including the administrative area. Among the improvements to be made in Delicias del Norte is the utilization of production capacity taking into account that it possesses all the necessary requirements to increase production. In the marketing process it is aimed to improve the marketing process through increased investment in advertisement material.
\end{abstract}

Keywords: Process, production, marketing. 


\section{INTRODUCCIÓN}

La presente investigación describe el proceso de producción de las rosquillas de Delicias del Norte y Caracteriza las estrategias de comercialización utilizadas por esta empresa. El centro de atención de este estudio es la producción y comercialización de rosquillas somoteñas de Delicias del Norte ya que estas cumplen con todos los requisitos para producir con calidad gracias a las Buenas Prácticas de Manufactura y se diferencian de las producidas en demás talleres de rosquillas por contar con su propio empaque y por la forma en que han venido innovando la producción de rosquillas.

Cuando hablamos de producción es la actividad de utilizar los procesos, máquinas y herramientas en conjunto con las operaciones mentales y manuales con el fin de obtener unos productos a partir de las materias y componentes básicos. (Juram J M,GrynaJrFranCm,Bingham,JrR.S, 2005, pág. 267)

En cuanto a comercialización implica el vender, dar carácter comercial a las actividades de mercadeo, desarrollar estrategias y técnicas de venta de los productos y servicios, la importación y exportación de productos, compra-venta de materia prima y mercancías al por mayor, almacenaje, la exhibición de los productos en mostradores, organizar y capacitar a la fuerza de ventas, pruebas de ventas, logística, compras, entregar y colocar el producto en las manos de los clientes, financiamiento etc. (James Jordan,, 2008)

Delicias del Norte, está ubicado al sur de la ciudad de Somoto Madriz. Esta es una empresa de la Agroindustria, fundada en el año 2005 por Flora Matea Ortiz una empresaria con gran experiencia en gestión y administración en la industria de derivados del maíz (Rosquillas) para el mercado regional, nacional e internacional.
La capacidad productiva de Delicias del Norte se ve limitada y no se aprovecha al máximo por una visión empresarial de poco riesgo por parte de la propietaria a pesar de contar con los requisitos necesarios para producir como: registros sanitarios, empaques, buenas prácticas de manufactura, personal capacitado y la diversificación de sus productos.

Otra de las limitantes es ampliar el número de clientes y de zona para comercializar las rosquillas en la ciudad de Somoto por el incremento de los competidores que se introducen en el mercado ya que la mayoría es competencia desleal.

Obtener los productos como el maíz, la leche y el queso es una dificultad porque son productos que por periodos son escasos, en la empresa cuentan con diversos productores de la zona que varían los precios de acuerdo a sus periodos de producción. Este tipo de materia prima no puede ser sustituida por productos de bajos precios porque influye en la calidad y durabilidad de sus rosquillas, esta situación eleva el costo de producción, baja las ganancias y se corre el riesgo de reducir el empleo, por las pérdidas económicas.

Otra de las dificultades observadas en la producción es que no cuenta con la infraestructura adecuada, ni materiales para almacenar el maíz, este es almacenado fuera de la empresa y se tiene que trasladar a diario lo que quita tiempo para agilizar el proceso productivo, tampoco almacena el dulce y la margarina que son necesarios para el proceso productivo y es por ellos compra únicamente la cantidad que se va a necesitar para la producción del día.

En el proceso de comercialización los mayores problemas se relacionan con el proceso de distribución por la falta de medios tales como los canales de distribución equipos e inversiones y la falta de personal encargado de la comercialización del producto. 


\section{La gráfica $N^{\circ}$ 2. Muestra el Flujo del Proceso de Producción}

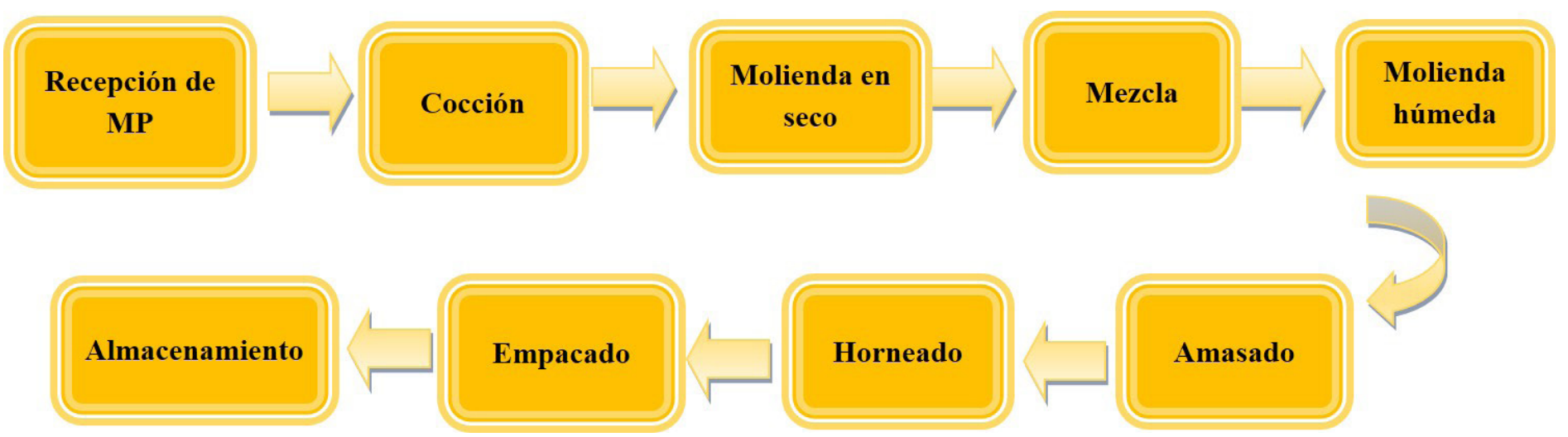

\section{Capacidad productiva y control de calidad}

Delicias del norte es una empresa constituida legalmente con una buena capacidad productiva ya que cuenta con todas las condiciones para producir e introducir sus productos en nuevos mercados pero esta capacidad productiva se ve limitada ya que la empresa se dedica a producir para satisfacer únicamente a los clientes fijos y no se arriesga a conquistar nuevos mercados. La planeación de la producción se hace de acuerdo a los pedidos pero ya se tienen establecidos algunos como por ejemplo:

- Producir diariamente un quintal y medio excepto una vez al mes que es cuando hay más pedidos ya que siendo así se producen dos quintales.

- Exportar cada mes un pedido a Costa Rica de 30 cajas que equivalen a 15 paquetes de rosquillas.

- Exportar cuatro cajas una vez al mes a España.

- Exportar a Estados Unidos tres cajas cada mes, esto se hace a personas particulares.

Durante el proceso productivo se toma en cuenta la higiene porque es una parte fundamental para la calidad del producto terminado, por eso existen reglas estrictas para laborar: los empleados no deben usar perfumes, cremas, aretes, collares, pulseras. También deben de usar gorros de maya para cubrirse el cabello así como gabachas, mascarillas y usar únicamente tenis.
Cuentan con una buena seguridad laboral ya que los trabajadores tienen garantizados sus equipos necesarios para laborar y se trabaja de manera artesanal, están certificados con el MINSA ya que este le entrego su licencia sanitaria, registro sanitario, código de barra y en buenas prácticas de manufactura (BPM) la que se obtuvo mediante un programa de limpieza desde la materia prima hasta el producto empacado.

"La aplicación de las BPM consiste en llevar un registro donde se anotan paso a paso todo lo que se va haciendo, a qué hora, quien lo hace y quien lo firma, es decir la que lo hizo lo llena y lo firma y yo lo superviso y lo firmo".

Al mismo tiempo que se aplican las BPM, se implementan la producción más limpia. Para eso se hizo un conteo de cuantas rosquillas salen de una libra de masa y el maíz para ponerlo a precoser, luego se pesa; cuando ya está la harina hay que pesarla de nuevo, para confirmar que la fórmula que se está usando es exacta siempre es decir tanto de harina tanto de queso tanto margarina. Cuando ya tiene todos los ingredientes tiene que dar otro peso para saber cuántas rosquillas de cada kilo.

\section{Costos de producción:}

Materia Prima: La materia prima necesaria para el proceso de producción de rosquillas Somoteñas es: el maíz, queso, leche, margarina, dulce de panela. La 
Gerencia: en el área de gerencia se encuentra el área de administración y finanzas dirigida por Flora Matea Ortiz con el cargo de gerente administrativa. Esta constituye una de las áreas más importante en Delicias del Norte la cual se encarga de llevar a cabo todo el proceso Administrativo y la toma de decisiones.

Dentro de esta misma área está también la contadora, Socorro López ella es la encargada de cumplir con las actividades propias de contabilidad destacándose entre ellas los registros contables, realización de compras de los insumos necesarios para la producción y el control de inventario.

Producción: Dentro de esta área se llevan a cabo las actividades vinculadas con el proceso productivo la encargada del área de producción es Meyling Balladares quien dirige a las obreras siguientes:

Las figuradoras: quienes moldean la masa y hacen las figuritas de rosquillas hojaldras palitos y empanadas dentro de esta área se encuentran: Raquel Sequeira, Flor Hernández, Oneyling Ponce, Adela Herrera. La endulzadora Vilma Balladares es la que se encarga de endulzar las hojaldras y todas ellas también se encargan de empacar las rosquillas para la venta.

Mantenimiento: en esta área se encuentra Jonys Alanís quien es encargado de dar mantenimiento a las áreas de producción de la empresa.

Ventas: el gerente de ventas es Ronaldo Ortiz es el encargado de llevar el control de las ventas y los pedidos que realizan los clientes y de él depende:

La encargada de ventas quien es la que implementa las normas de control de personal y las normas de calidad del producto, es también la encargada del pago de nómina y de control de expediente de cada uno de los expedientes.

\section{Proceso de producción}

Maquinaria: La empresa cuenta con dos molinos semi-industriales los cuales funcionan a base de electricidad, un termostato que funciona con batería para controlar la temperatura de los hornos y una selladora para el empaque de las rosquillas la cual funciona con electricidad.

Mano de obra directa: Para la elaboración de las rosquillas la empresa cuenta con 10 obreros cada uno realiza diferentes funciones que van desde la selección de la materia prima hasta el almacenamiento del producto terminado. En Delicias del Norte se produce 1 quintal y medio de maíz los empleados labora por la mañana de 6 de la mañana a 2 de la tarde.

Infraestructura: La empresa cuenta con una área de limpieza y molienda, una área de amasado y figurado, una área para el horneado y una área de empaque así como el área de oficinas de administración.

Proceso de conversión: El proceso de conversión que utiliza la empresa Delicias del Norte es semi-industrial por que utilizan una combinación del proceso artesanal y utilizan también molinos eléctricos y hornos con termostato 
Delicias del Norte no cuenta con una estrategia de comercialización que les permita introducir sus productos en nuevos mercados nacionales. La distribución de las rosquillas somoteñas se ha venido centralizando en un mercado diferenciado de consumidores nostálgicos que viven en el extranjero como en Costa Rica, Chile, Estados Unidos y Venezuela. La empresa cuenta con empaques para algunos de sus productos como las rosquillas, hojaldras y palitos con chile, pero los demás productos que ha venido innovando aun no tienen su propia imagen para introducirse en el mercado. Otra dificultad es la poca inversión en el material publicitario, solo se da a conocer en ferias y la red social de Facebook y no utiliza medios de comunicación (radio o televisión) para divulgar sus productos, características y calidad.

Esta investigación será de utilidad para Delicias del Norte por que permite la identificación del proceso de producción y comercialización enfocándose en sus dificultades y debilidades a fin de hacer una propuesta de estrategia que mejore el proceso de comercialización de las rosquillas. Así mismo, este documento será de utilidad para las instituciones u organismos que asesoran a las micro y pequeñas empresas dedicadas a la producción de rosquillas, para que promuevan acciones que fortalezcan su proceso de producción y estrategias de comercialización

\section{MATERIALES Y MÉTODOS}

Esta investigación es aplicada, ya que se propone una estrategia de comercialización para la empresa de rosquillas somoteñas Delicias del norte. Además es de tipo cualitativa por que se describe y analiza a profundidad el proceso de producción y comercialización de las rosquillas.

El tipo de muestreo es no probabilístico porque la población de estudio es pequeña. Dentro de los criterios de selección de la muestra están: 1) Participación voluntaria en el estudio, 2) Conocimiento sobre el tema de investigación, 3) Disposición de los trabajadores para brindar información.

Se utilizaron tres técnicas de recolección de datos: la entrevista, la guía de observación y la investigación documental. Las entrevistas se aplicaron a las personas más accesibles y que estaban involucradas directamente con el proceso de producción y comercialización de las rosquillas en especial a la propietaria de la empresa, al encargado del área de producción así como al encargado del área de ventas, y al encargado de la distribución de este producto.

La observación se realizó en dos áreas de la empresa primero se tomó en cuenta todo lo que se hacía en el área de producción para observar tanto el proceso de elaboración de las rosquillas como el cumplimiento de los estándares de calidad, también se observó el área de comercialización de las rosquillas para observar si cumple con los requisitos para comercializar.

\section{RESULTADOS Y CONCLUSION:}

Descripción de la empresa Delicias del Norte en la ciudad de Somoto Madriz.

Doña Florita como la llaman cariñosamente es la propietaria de Delicias del Norte la cual empezó en el año 2005 a producir rosquillas de manera familiar con su mama y sus hermanos, horneando una arroba de maíz a la semana que equivale a 25 libras.

En el año 2006 conoció el programa ProEmpresa quien la apoyó con financiamiento y asistió a todas las reuniones y capacitaciones que organizaban pues tenía todo el deseo de iniciar su empresa cumpliendo con todos los requisitos y normas para producir, estas actividades eran realizadas una vez por semana con un grupo asociativo que tenían con personas de Somoto, Yalaguina y la Esperanza. También fue apoyada con financiamiento en cuanto a la infraestructura, código de barra y registro sanitario por el mismo programa y organizaciones como el IDR. 
Luego la apoyo voces vitales donde aprendió las herramientas teóricas y prácticas para engrandecer su empresa.

Esta empresa inicio con tres trabajadores. El Ministerio de Salud (MINSA), le entrego su licencia sanitaria, registro sanitario, código de barra, y hasta eligieron a su dueña como presidenta del primer grupo asociativo de las empresarias de rosquillas de Somoto en la cooperativa multisectorial "GERSON R.L" que nació en el año 2008 con el apoyo de PROEMPRESA con el fin de mejorar la posición competitiva de la cadena de valor de las rosquillas Somoteñas la cual está integrada por 56 socios.

Se dedica a la producción de rosquillas en diversas presentaciones: hojaldras, rosquillas, hojaldras rellenas, palitos picantes y empanaditas (quesadillas).

Desde sus comienzos su propietaria ha mostrado el interés de mejorar la empresa por lo que ha llevado a cabo cambios importantes entre los cuales está el cambio de hornos artesanales por hornos artesanales mejorados, con el fin disminuir el consumo de leña y contribuir al cuido del medio ambiente a través del uso de la poda de los árboles.

La propietaria de esta empresa ha optado por romper lo tradicional y aventurarse en el uso de otros tipos de dulces, como por ejemplo mermeladas de guayaba en las hojaldras. También está preparando una fórmula para empezar a vender rosquillas dietéticas, cuyo mercado será para personas obesas, diabéticas, entre otras. "Es una materia prima más baja de grasas, más baja de calorías".

Misión: Producir el mejor producto del mercado, bajo estrictas normas de higiene, calidad e inocuidad que garanticen la salud y satisfacción de nuestros clientes de los mercados nacionales e internacionales.

Visión: Ser una empresa modelo a nivel nacional cubriendo demandas de mercados internacionales siempre apegados a los cuatro pilares de la empresa: Tradición, calidad, conciencia ambiental e innovación.

\section{Grafica $N^{\circ} 1$. Estructura organizativa:}

\section{Funciones de los cargos:}

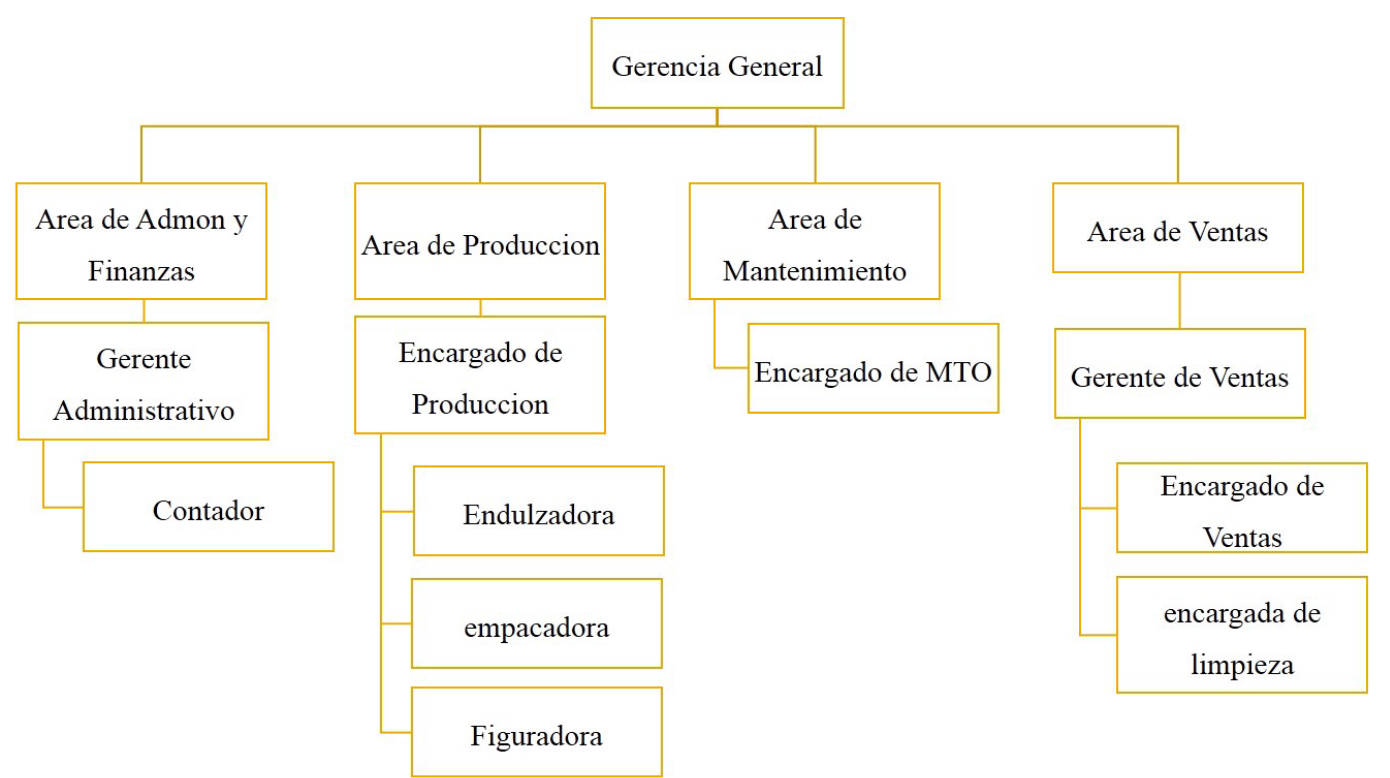


materia prima principal es el Maíz. Los principales proveedores de materia prima son los productores a quienes se los compran de manera directa en el caso del maíz que es el más importante.

\section{Escases y altos precios de Materia Prima}

"El escases y altos precios de la materia afectan directamente el proceso productivo de Delicias del Norte, pero se está buscando la forma de solucionar este problema por medio de capacitaciones para la adaptación a los cambios climáticos."

En el cuadro número 1 se representa la comparación de los costos de materia prima entre el año 2013 y 2014. El maíz se compra de manera semestral.

\begin{tabular}{|l|l|l|l|l|}
\hline \multicolumn{5}{|c|}{ Comparación de precios del maíz año 2013-2014 } \\
\hline \multicolumn{1}{|c|}{ Periodos } & $\begin{array}{l}1^{\text {er }} \text { semestre } \\
2013\end{array}$ & $\begin{array}{l}2^{\text {do }} \text { semestre } \\
2013\end{array}$ & $\begin{array}{l}1^{\text {er }} \text { semestre } \\
2014\end{array}$ & $\begin{array}{l}2^{\text {do }} \text { semestre } \\
2014\end{array}$ \\
\hline Cantidad qq & 156 & 156 & 234 & 234 \\
\hline $\begin{array}{l}\text { Precios } \\
\text { Unitarios }\end{array}$ & $\mathrm{C} \$ 300.00$ & $\mathrm{C} \$ 380.00$ & $\mathrm{C} \$ 400.00$ & $\mathrm{C} \$ 500.00$ \\
\hline Precio Total & $\mathrm{C} \$ 46800.00$ & $\mathrm{C} \$ 59280.00$ & $\mathrm{C} \$ 93600.00$ & $\mathrm{C} \$ 117000.00$ \\
\hline
\end{tabular}

El cuadro numero 2 representa las utilidades obtenidas de la producción tanto para el mercado nacional como extranjero.

\begin{tabular}{|l|l|l|}
\hline \multicolumn{3}{|c|}{ Cuadro de utilidad } \\
\hline Concepto & C\$ & C\$ \\
\hline Ventas Netas & & $460,350.00$ \\
\hline Costos Variables & $265,926.00$ & \\
\hline Costos Fijos & 3260.00 & \\
\hline Costo Total & & $269,186.00$ \\
\hline Utilidad & & $191,164.00$ \\
\hline
\end{tabular}

\section{Proceso de comercialización}

Delicias del Norte no cuenta con una persona encargada de mercadeo de sus productos que se encargue de buscar nuevos mercados a nivel nacional y que se encargue de plantear nuevas estrategias que garanticen la ampliación y comercialización de las rosquillas. particulares. diario de maíz".
Las rosquillas que producen "Delicias del Norte" son comercializadas de manera directa ofreciendo sus productos en las propias instalaciones de la empresa aunque también se realizan entregas por encomiendas a los diferentes departamentos de Nicaragua para el consumo nacional, las exportaciones también son directas a un comprador particular en el extranjero quien se encarga de la distribución por su propia cuenta el cual trabaja de forma independiente a la empresa.

\section{Delicia del Norte}

Consumidor final

También vende por medio de canales de distribución ya que los pedidos de supermercados y otros puntos de venta se atiende a través de un distribuidor nacional quien se encarga de poner el producto en las manos de quien hace el pedido garantizando la entrega del producto en buen estado.

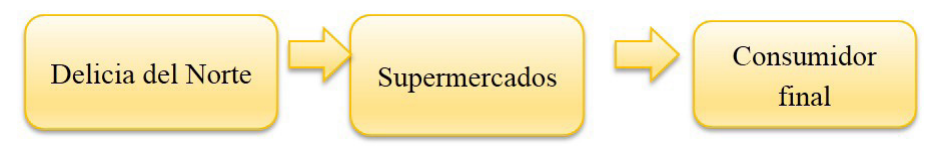

Los principales compradores a nivel nacional son las gasolineras y los supermercados especialmente en león y granada, y a nivel internacional son enviados a Costa Rica, Estados Unidos, y España a personas

\section{Estrategia de Comercialización Utilizada por Delicias del Norte}

"Contamos con un plan estratégico para tres años donde se define la producción de un quintal y medio 
La mayoría de la publicidad que utilizan es impresa en tarjetas de presentación que entregan a sus visitantes, $\mathrm{y}$ en las etiquetas que usan en el empaque. Actualmente, han diseñado una página en la red social Facebook. Participan activamente en ferias locales y nacionales. El transporte para comercializar los productos está pintado con publicidad de la empresa.

Los precios de venta son establecidos según el tipo de clientes. A quienes les compran una vez al mes se les vende la bolsa a 20 córdobas, a los que compran más de una vez al mes se les vende a 18 córdobas y al distribuidor, que es uno de los máximo compradores, se le da a un precio preferencial de 17 córdobas.

Esta pequeña empresa se ha caracterizado por la innovación de sus productos por eso cada día intenta sorprender al mercado. De hecho fue lo que la impulso a preparar nuevos productos como: Palitos con chile, bastones y empanadas dulce. La propietaria de esta empresa ha optado por romper lo tradicional y aventurarse en el uso de otros tipos de dulce, como: mermeladas de guayaba y de coco en las hojaldras.

También está preparando una fórmula para vender rosquillas dietéticas, cuyo mercado será para personas que cuidan su dieta alimenticia, "es una materia prima más baja en grasa y calorías". Sin embargo, estos productos aún no se comercializan en el mercado per que el empaque es una de las limitantes para el proceso de comercialización ya que no todos los productos que esta empresa elabora cuentan con empaques porque en Nicaragua no elaboran el empaque con el material requerido para garantizar la calidad y durabilidad de las rosquillas y tienen que importar los empaques de Honduras lo que incrementa los costos y por consiguiente aumenta el valor agregado del producto.

El cuadro $\mathrm{N}^{\circ} 3$ muestra las ventas mensuales y anuales de Delicias del Norte

\begin{tabular}{|l|l|l|l|l|}
\hline \multicolumn{5}{|c|}{ Unidades vendidas } \\
\hline Descripción & Unidades & Venta Diaria & venta Mensual & venta Anual \\
\hline Mercado nacional & Bolsas & 900 & 23,400 & 280,800 \\
\hline exportaciones & Bolsas & 1200 & 1200 & 14,400 \\
\hline \multicolumn{2}{|c|}{ Total } & $\mathbf{2 1 0 0}$ & $\mathbf{2 4 , 6 0 0}$ & $\mathbf{2 9 5 , 2 0 0}$ \\
\hline
\end{tabular}

\section{Propuesta de estrategia de comercialización:}

\section{Análisis FODA del proceso de comercialización:}

\begin{tabular}{|c|c|}
\hline Fortalezas & Oportunidades \\
\hline $\begin{array}{l}\text { - } \text { Aceptación de las rosquillas por los clientes nacionales } \\
\text { - } \quad \text { experinjeroncia en producción de rosquillas. } \\
\text { - } \text { Oferta variada de rosquillas } \\
\text { - } \text { Implementación de buenas prácticas de manufactura } \\
\text { (BPM). } \\
\text { - Mejora en el proceso de producción mediante } \\
\text { producción más limpia. } \\
\text { - } \text { Reconocimiento de la empresa por los logros } \\
\text { obtenidos a través de diferentes certificados. } \\
\text { - Capacitaciones continúas del MINSA. } \\
\text { Cuentan con registro sanitario actualizado, código de } \\
\text { barra y empaque. } \\
\text { Empresa constituida legalmente. }\end{array}$ & $\begin{array}{l}\text { - } \quad \text { Exportación de sus productos al extranjero. } \\
\text { - } \quad \text { Certificación de buenas prácticas de manufactura por } \\
\text { el Minsa. } \\
\text { - Capacitación para la adaptación a los cambios } \\
\text { climáticos. } \\
\text { - Perteneciente en cooperativa Gerson RL. }\end{array}$ \\
\hline
\end{tabular}




\begin{tabular}{|l|l|}
\hline Debilidades & Amenazas \\
\hline $\begin{array}{l}\text { - Visión empresarial de poco riesgo. } \\
\text { - La infraestructura no tiene las condiciones para } \\
\text { almacenar materia prima e insumos. }\end{array}$ & $\begin{array}{l}\text { Competencia desleal de talleres artesanales de rosquillas } \\
\text { en la zona. } \\
\text { Variación de costos de la materia prima (leche y queso) } \\
\text { en periodos de escases (verano). }\end{array}$ \\
- Escasez y alto precio de la materia prima. & \\
- No cuenta con una estrategia publicidad. & \\
- Falta de medios y recursos para distribuir las rosquillas. & \\
- $\quad \begin{array}{l}\text { Fa importación del empaque. } \\
\text { intermediarios en el extranjero. }\end{array}$ & \\
\hline
\end{tabular}

\section{Líneas estratégicas para mejorar las estrategias de comercialización de rosquillas somoteñas en Delicias del Norte.}

En base al análisis FODA realizado anteriormente tomando en cuenta las mayores debilidades de la empresa Delicias del Norte se proponen a continuación las siguientes estrategias para mejorar el proceso producción y comercialización de las rosquillas:

Líneas $\mathbf{N}^{\mathbf{0}}$ 1: Aprovechar la capacidad productiva actual de la empresa.

Objetivo: Incrementar la capacidad de oferta en nuevos mercados.

Líneas $N^{\mathbf{0}}$ 2: Ampliar la infraestructura actual de la empresa.

Objetivo: Mejorar la capacidad de almacenamiento mediante la remodelación de las áreas y las instalaciones.

Líneas $N^{\mathbf{0}}$ 3: Mejorar el proceso de comercialización. Objetivo: Ampliar la Distribución de las Rosquillas de Delicias del Norte

Líneas $N^{0}$ 4: Diseñar una estrategia de publicidad.

Objetivo: Divulgar las características y los beneficios de la calidad de las rosquillas en el mercado.

Líneas No 5: Mejorar la presentación del producto.

Objetivo: Comercializar los productos que aún no cuentan con empaque para atacar la competencia desleal.

Líneas $\mathbf{N}^{0}$ 6: Realizar promociones de ventas.

Objetivo: Establecer nuevos mercados dentro del público consumidor y aumentar su volumen de ventas.

\section{CONCLUSIONES}

Al finalizar el presente estudio investigativo en la empresa de Rosquillas Somoteñas Delicias del Norte podemos concluir en base a los objetivos específicos planteados lo siguiente:

Es de vital importancia que las empresas mejoren su proceso de producción y comercialización de las rosquillas Somoteñas y Delicias del Norte no es la exención y a pesar de que ha venido desarrollándose lentamente su proceso productivo se ha venido mejorando ya que paso de ser una empresa familiar a una empresa que genera ingresos a muchas mujeres del municipio de Somoto.

Esta pequeña empresa en su proceso productivo trabaja con estándares de calidad que han venido mejorando mediante la planeación de sus operaciones y el control adecuado de inventario tanto para su materia prima como para sus productos terminados.

En el proceso productivo de esta empresa nos encontramos con un proceso de producción bien 
organizado y con el cumplimiento de todas las normas y la implementación de las buenas prácticas de manufactura (BPM) durante el proceso de producción de las rosquillas. De igual forma se encuentran una serie de limitantes: como la escasez y los altos precios de la materia prima y porque a pesar de contar con todos los elementos necesarios para producir no se arriesga a introducir sus productos en diferentes mercados.

Durante la investigación y el análisis que se realizó en el proceso de comercialización de las rosquillas somoteñas de Delicias del Norte se observó que esta empresa así como ha venido aumentando aunque de manera lenta la cantidad de productos así también ha venido incrementando la demanda de sus productos pero aún falta mucho por hacer porque la mayoría de estos clientes son clientes que compran poco y les compran de manera directa. Ellos dentro de la empresa no tienen una persona encargada de mercadeo, ni una persona que se encargue de la distribución de los productos.
Mediante el estudio también se concluye que las rosquillas tienen una buena aceptación por los clientes fijos tanto a nivel nacional como internacional. Pero esta aceptación se ve limitada para conquistar nuevos clientes por qué no invierten en material publicitario.

En resumes se puede decir que a pesar de que existen muchas barreras también existen grandes oportunidades en el proceso de producción y comercialización que ayudaran al desarrollo y crecimiento de esta empresa.

\section{BIBLIOGRAFIA}

Juram.J.M, Gryna Jr FranCm, Bingham, Jr R.S (2005). Manual de control de calidad, volumen I, Segunda Edición. pág. 267.

James Jordan. ¿Qué es comercialización? Pág. http://www.tumercadeo.com/2010/02/que-escomercializacion.html. Fecha de consulta 05 de obtubre 2014 